\title{
PALM-3000: EXOPLANET ADAPTIVE OPTICS FOR THE 5 m HALE TELESCOPE
}

\author{
Richard DekANY ${ }^{1}$, Jennifer Roberts ${ }^{2}$, Rick Burruss ${ }^{2}$, Antonin Bouchez $^{1,3}$, Tuan Truong ${ }^{2}$, Christoph Baranec $^{1}$, \\ Stephen Guiwits $^{2}$, David Hale ${ }^{1}$, John Angione ${ }^{2}$, Thang Trinh ${ }^{2}$, JeFFry Zolkower ${ }^{1,4}$, J. Christopher Shelton ${ }^{2}$, \\ Dean Palmer $^{2}$, John Henning ${ }^{1}$, Ernest Croner ${ }^{1}$, Mitchell Troy $^{2}$, Dan McKenna $^{1}$, Jonathan Tesch $^{2}$, \\ Sergi Hildebrandt ${ }^{1,2}$, And Jennifer Milburn ${ }^{1}$ \\ ${ }^{1}$ Caltech Optical Observatories, California Institute of Technology, 1200 East California Boulevard, MC 11-17, Pasadena, CA 91125, USA; rgd@ astro.caltech.edu \\ 2 Jet Propulsion Laboratory, California Institute of Technology, 4800 Oak Grove Boulevard, Pasadena, CA 91109, USA \\ Received 2013 June 5; accepted 2013 August 19; published 2013 October 7
}

\begin{abstract}
We describe and report first results from PALM-3000, the second-generation astronomical adaptive optics (AO) facility for the $5.1 \mathrm{~m}$ Hale telescope at Palomar Observatory. PALM-3000 has been engineered for high-contrast imaging and emission spectroscopy of brown dwarfs and large planetary mass bodies at near-infrared wavelengths around bright stars, but also supports general natural guide star use to $V \approx 17$. Using its unique $66 \times 66$ actuator deformable mirror, PALM-3000 has thus far demonstrated residual wavefront errors of $141 \mathrm{~nm}$ rms under $\sim 1^{\prime \prime}$ seeing conditions. PALM-3000 can provide phase conjugation correction over a 6.'4 $\times 6.4$ working region at $\lambda=2.2 \mu \mathrm{m}$, or full electric field (amplitude and phase) correction over approximately one-half of this field. With optimized back-end instrumentation, PALM-3000 is designed to enable $10^{-7}$ contrast at $1^{\prime \prime}$ angular separation, including post-observation speckle suppression processing. While continued optimization of the AO system is ongoing, we have already successfully commissioned five back-end instruments and begun a major exoplanet characterization survey, Project 1640.
\end{abstract}

Key words: atmospheric effects - instrumentation: adaptive optics - minor planets, asteroids: individual (Ganymede) - planets and satellites: detection - techniques: high angular resolution - techniques: imaging spectroscopy

Online-only material: color figures

\section{INTRODUCTION}

Adaptive optics (AO) systems have proven essential in opening key new areas of astronomical research by compensating for atmospheric blurring to achieve diffraction-limited infrared imaging and spectroscopy. The successful development of AO capabilities on the world's large-aperture telescopes over the past two decades is a scientific achievement of a large and dedicated instrumentation community (Hart 2010; Davies \& Kasper 2012). The Palomar Adaptive Optics System (PALM-241) on the $5.1 \mathrm{~m}$ Hale telescope at Palomar Mountain (Dekany et al. 1997; Troy et al. 2000; Dekany et al. 2006) has been a leader in astronomical AO, providing facility-class on-sky operation since 1999 December. The instrument, jointly developed by Jet Propulsion Laboratory and Caltech, was used to obtain data contributing to $\approx 100$ refereed astronomical journal articles while facilitating extensive AO technology development. The advantages of accessible site, moderate altitude, and excellent engineering support infrastructure have supported the on-sky deployment of many innovations: the first high-speed AO telemetry recording system (Truong et al. 2003), the now widespread SciMeasure high-speed wavefront sensor camera (DuVarney et al. 2000a, 2000b, 2001), the first high-order natural guide star (NGS) Shack-Hartmann simultaneous multiple star wavefront sensor (Velur et al. 2006), demonstrations of sparse matrix (Shi et al. 2003), hierarchical (MacMartin 2003), efficient recursive (Ren et al. 2005) and Fourier transform based slope sensor reconstructors (Poyneer et al. 2003), AO-assisted "lucky" imaging at visible wavelengths (Law et al. 2009), vector-vortex coronagraph (Serabyn et al. 2009), non-redundant mask interferometry

\footnotetext{
3 Now at Giant Magellan Telescope Observatory Corporation, Pasadena, CA 91106, USA.

4 Now at Department of Astronomy, Cornell University, Ithaca, NY 14853, USA.
}

(Bernat et al. 2010), and three years of solid-state sum-frequency $589 \mathrm{~nm}$ laser guide star operations (Velur et al. 2004; Bouchez et al. 2007; Roberts et al. 2008) including the demonstration of back pumping and frequency chirping techniques that increase the efficiency of photoreturn from Earth's mesospheric sodium layer (Kibblewhite 2008, 2009). PALM-241 achieved infrared $K$-band imaging Strehl ratios under median seeing conditions of 1"1. as high as $73 \%$ as measured using point-spread function (PSF) fitting photometry (Roberts et al. 2004). Exposures of at least $30 \mathrm{~s}$ are needed with our instrumentation to estimate infrared Strehl ratios properly, accounting for the faint, extended contribution of the PSF halo. Assuming Marechal's approximation (Marechal 1947), this is an equivalent rms wavefront error of $195 \mathrm{~nm}$. Using a centrally projected guide star laser emitting up to $8 \mathrm{~W}$ of $589 \mathrm{~nm}$ sodium $D_{2}$ light to form a mesospheric synthetic beacon, PALM-241 achieved $48 \% K$-band imaging Strehl as part of a laser proof-of-concept demonstration science program (Roberts et al. 2008).

Since the initial discovery of a confirmed substellar object at Palomar Observatory (Nakajima et al. 1995), low-mass objects have been a focus of direct imaging searches and have motivated the Palomar AO program. Despite excellent performance and successful investigations of brown dwarf companions (Boccaletti et al. 2003; Lloyd et al. 2006; González-García et al. 2006; Metchev \& Hillenbrand 2009), the ability of PALM-241 to directly image stellar companion exoplanets using the full $5.1 \mathrm{~m}$ aperture was limited to a contrast of $\sim 10^{-4}$ at 0.5 by imperfect complex amplitude correction of the electromagnetic field at the coronagraphic occulting mask, resulting in residual semi-static stellar speckles (Bloemhof et al. 2000a; Oppenheimer et al. 2000; Bloemhof et al. 2001). These speckles would vary in amplitude as well as contribute photon noise in the focal plane. Improving exoplanet imaging capabilities beyond these limitations, to approach the theoretical limits for 
ground-based exoplanet study (Angel 1994), required a combination of improved calibration, post-processing techniques, and capability of the AO system.

PALM-3000 is the second-generation astronomical AO system for the Hale telescope. It has been engineered specifically to optimize high-contrast studies of exoplanets orbiting nearby bright stars when used in conjunction with specialized specklesuppressing instrumentation, including Project 1640 (P1640; Hinkley et al. 2011; Zimmerman et al. 2011), the Palomar HighAngular Resolution Observer (PHARO; Hayward et al. 2001), and the Palomar Fiber Nuller (PFN; Hanot et al. 2011). The combination of PALM-3000 and P1640, in particular, is expected to reach $5 \sigma$ detection contrast ratios as high as $10^{-7}$ at an angular radius of $1^{\prime \prime} .0$ and $10^{-4}$ at 0 '. 3 after application of speckle suppression algorithms exploiting P1640's coherent integral field spectroscopic data cube.

To date, our team has conducted commissioning observations with PALM-3000 and five visible and near-infrared science instruments. In particular, we have quantified the benefit of the speckle suppression technique using P1640, in terms of the faintest companion that can be detected with $5 \sigma$ confidence as a function of separation from a star. Initial observations achieved a contrast of $10^{-5}$ at $0^{\prime \prime} .5$ angular separation from an occulted star (Oppenheimer et al. 2012), suitable for detecting luminous substellar companions, though our integrated system is not yet at full performance awaiting improved instrument calibration and $\mathrm{AO}$ improvements. Future studies will report on the results of our optimization activities.

\section{SCIENCE MOTIVATION}

\subsection{Exoplanet Spectroscopy}

The direct imaging discovery of planetary mass companions to HR 8799 (Marois et al. 2008) and Fomalhaut (Kalas et al. 2008) with orbital radii of tens of AU has inaugurated a new era in the study of exoplanets. Radial-velocity and transit detection techniques, so fruitful for exoplanet discovery, are currently only sensitive to detection of a strongly irradiated class of planets orbiting close in to their host stars. Fully mature highcontrast direct imaging campaigns, on the other hand, will be sensitive to companions at large separations and will probe a critical region of the exoplanetary parameter space. Direct imaging studies will allow us to address questions about solar system architectures by evaluating the frequency of companions undetectable by radial-velocity and transit methods. Even a flat extrapolation of the semi-major axis distribution of radialvelocity detected exoplanets beyond 3-5 AU foreshadows a large population of undetected planets around nearby stars (Crepp \& Johnson 2011). In addition, the increasing frequency of planet masses discovered through radial-velocity techniques below about $4 M_{\text {Jup }}$ suggests a large population of lower mass planets exist around nearby stars. Moreover, high-contrast imaging campaigns will further constrain our understanding of the distribution of brown dwarf masses and their frequency around a variety of host stars. High-contrast integral field spectrographs will allow study of massive exoplanets in detail, tackling questions about their internal physics, structure, and atmospheric chemistry.

With our partners (Hinkley et al. 2011) we have designed a survey to search for and characterize exoplanets during 99 nights of dedicated observing with PALM-3000 and P1640. Motivated by the correlation between planet frequency and the mass of the host star (Johnson et al. 2007), the survey is targeting approximately 220 stellar systems having A- and F-type stars, a population expected to host exoplanets five times as often as their lower mass counterparts. Indeed, two of the first imaged exoplanet systems, HR 8799 and Fomalhaut, have A-type hosts. A secondary survey will also be performed of stars within 25 pc with visual magnitude $V<7$ establishing a complete census of wide-orbit candidates. Already, PALM-3000 and the P1640 instrument have obtained simultaneous infrared spectra of the HR 8799 giant planets (Oppenheimer et al. 2013), moving exoplanet study into a spectrographic science. With the expected final AO performance of PALM-3000 (Section 5.1) we will be able to observe upward of $90 \%$ of the planets of greater than seven Jupiter masses orbiting the stars in our sample (Hinkley et al. 2011; Beichman et al. 2010). This survey will comprehensively determine whether the theorized brown dwarf "desert" (Kraus et al. 2008) exists. Depending on the true fraction of stars harboring exoplanets, we will additionally obtain low-resolution spectra of several planet-mass objects in each of several distinctly different age bins.

\subsection{Circumstellar Disks}

A comprehensive picture of the formation of planets can only be obtained with a thorough understanding of the structure and evolution of the circumstellar disks from which they evolve. After 5-10 Myr, most of the gas content of the primordial, massive and optically thick circumstellar disk has been cleared away (Haisch et al. 2001), accreted onto the star itself, formed into planetary bodies, or dispersed via stellar radiation. Stars exiting this primordial phase possess a much less massive optically thin disk, mostly containing second-generation grains, formed by the collisional fragmentation of larger bodies. IRAS and Spitzer have detected hundreds of debris disks by their far-infrared excesses (Wyatt 2008), but only a dozen images of optically thin disks are currently available (see circumstellardisks.org for an up-to-date list). Multi-wavelength resolved imaging is a necessary complement to the infrared spectral energy distribution analysis to remove degeneracies between grain morphology, temperature distribution, and grain size. The range and values of these parameters frame the conditions for planet formation.

Furthermore, the most prominent disk structures (e.g., warp, annulus, asymmetries) match the predicted dynamical effects based on the characteristics of embedded planets (Wyatt et al. 1999; Chiang et al. 2009; Chauvin et al. 2012). The Hubble Space Telescope has been a prime observatory for high-contrast imaging at short wavelengths $(\lambda=0.5-2.4 \mu \mathrm{m})$ for a decade, thanks to the Advanced Camera for Surveys (ACS) and NICMOS coronagraphs despite the large radii of their coronagraphic obscurations, i.e., large inner working angle (IWA), 0 .'9 for ACS, 0.5 for NICMOS. Unfortunately these two exceptional instruments are no longer in service. The James Webb Space Telescope will provide only modest short wavelength capabilities, as it will not be diffraction limited in the short near-IR and red visible bands unlike PALM-3000.

Using both P1640 (simultaneously recording both $J$ - and $H$-band spectra) and PHARO (providing $K$-band imagery), PALM-3000 will look across $\lambda=1.2-2.5 \mu \mathrm{m}$ at known and yetto-be identified spatially resolved debris disks at small IWA's, where warmer dust is likely to reside and where rocky planet formation is likely to occur (1-10 AU). Imaging such spatial scales is necessary to understand the interaction between the debris disk and planetary bodies, as well as the implications for planetary formation mechanisms. PALM-3000 will also characterize bright new candidates from the latest Spitzer and 


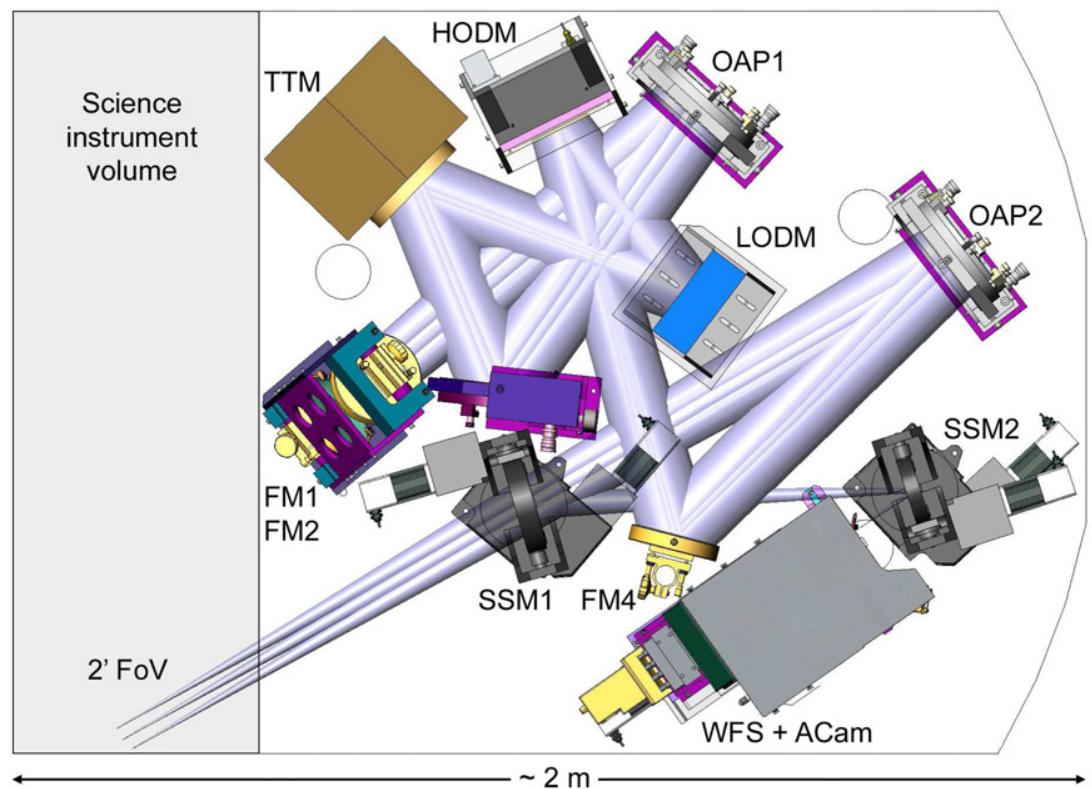

Figure 1. PALM-3000 optical layout, viewed from beneath the Cassegrain cage. Light passing through the PALM-3000 optical bench is folded by path-reducing fold mirrors, FM1 and FM2, to the collimating parabola, OAP1. In collimated space, the beam is folded by FM3 to the active tip-tilt mirror, TTM, followed by the low-order (LODM) and high-order (HODM) deformable mirrors, the latter of which resides conjugate to the entrance pupil (the telescope primary is the aperture stop). A fourth fold, FM4, sends light directly to a matched, re-imaging OAP2. In converging space, wavefront sensing light is split by an exchangeable dichroic/beam-splitter in reflection at the SSM1 star selection mirror, making a periscope pair with the complementary SSM2, before entering the focusable wavefront sensor, WFS, and acquisition camera assembly, ACam. Science light proceeds in transmission from SSM1 into the science instrument volume, where each back-end science instrument implements additional optics as necessary.

(A color version of this figure is available in the online journal.)

Herschel surveys. The sensitivity in $H$ and $K$ bands will be background-limited to $17-16 \mathrm{mag} \operatorname{arcsec}^{-2}$, respectively, which will allow imaging of brighter circumstellar disks around young stars, those having a ratio of infrared luminosity relative to their host star $(\mathrm{LIR} / L *)>10^{-3}$ at high signal-to-noise ratio.

\subsection{Well-corrected Subaperture Mode Inside 200 mas}

PALM-3000 retains the unique off-axis subaperture mode of PALM-241 (Serabyn et al. 2009). When used with the visible fast-frame imaging camera TMAS (Section 4) and a vector-vortex coronagraphic mask (Mawet et al. 2010), it will be possible to obtain high-contrast visible-light images of circumstellar material for guide stars of $V<2.5$. This highcontrast parameter space at extremely small IWA is largely unexplored (Mawet et al. 2012, 2013) and may include the possibility of detecting the first exoplanet in reflected light (Garrel et al. 2011). Simulations of this mode using the waveoptics propagation software PAOLA (Jolissaint et al. 2006) indicate an $R$-band Strehl ratio of $>90 \%$ is possible in good seeing conditions at Palomar, far exceeding reported visiblelight coronagraphic rejection to date (Swartzlander et al. 2008). Because the Monte Carlo based models of the frequency and distribution of exoplanets indicates the total number of detectable reflection exoplanets will be very limited (McBride et al. 2011), this mode is best considered a technique for targeted studies of known objects identified through radial velocity or transit photometry discovery. Similarly, contrast of better than $10^{-5}$ at an angular separation less than 0 .'2 has exciting possibilities for new studies of stellar astrophysics such as direct imaging of Herbig-Halo outflows (Bloemhof et al. 2000b; Femenía Castellá et al. 2010), Mira stars (Lagadec et al. 2011), and supergiant environments (Haubois et al. 2009).

\section{SYSTEM DESCRIPTION}

\subsection{Optical Design}

PALM-3000 uses the same pair of matched PALM-241 off-axis parabolic (OAP) mirrors to retain a compact one-toone magnification optical relay (Dekany \& Holm 1989). This economical design was adopted because of the need to reuse the PALM-241 deformable mirror (DM) within PALM-3000 (Section 3.2) and to respect the volume constraints within the Cassegrain cage environment of the Hale telescope. These OAPs were specified with $\lambda / 20$ peak-to-valley surface quality over $90 \%$ of their clear aperture and have been recoated without refiguring twice since 1997. Reuse of the OAPs also retains the same $112 \mathrm{~mm}$ diameter internal relay pupil. The optical layout and a description of the PALM-3000 optical paths is shown in Figure 1.

\subsection{Deformable Mirrors}

PALM-3000 uses two Xinetics, Inc. DMs sequentially within a collimated optical space to apply appropriate optical phase corrections. This architectural choice was necessitated by the relatively low $1.06 \mu \mathrm{m}$ actuator stroke of the dense highorder deformable mirror (HODM) relative to the turbulenceinduced requirement for total surface stroke $( \pm 5 \sigma)$ of $4.1 \mu \mathrm{m}$ in conditions where $r_{0}=9.2 \mathrm{~cm}$ (Hardy 1998) at $\lambda=500 \mathrm{~nm}$, corresponding to seeing of 1". 12 FWHM. The combination of high-spatial-frequency, small-stroke correction from the HODM and lower-spatial-frequency, larger-stroke correction from the low-order deformable mirror (LODM) allows PALM-3000 to correct most atmospheric conditions observed at Palomar Mountain (in the worst seeing conditions, the finite total mirror stroke available eventually limits correction capability.) 


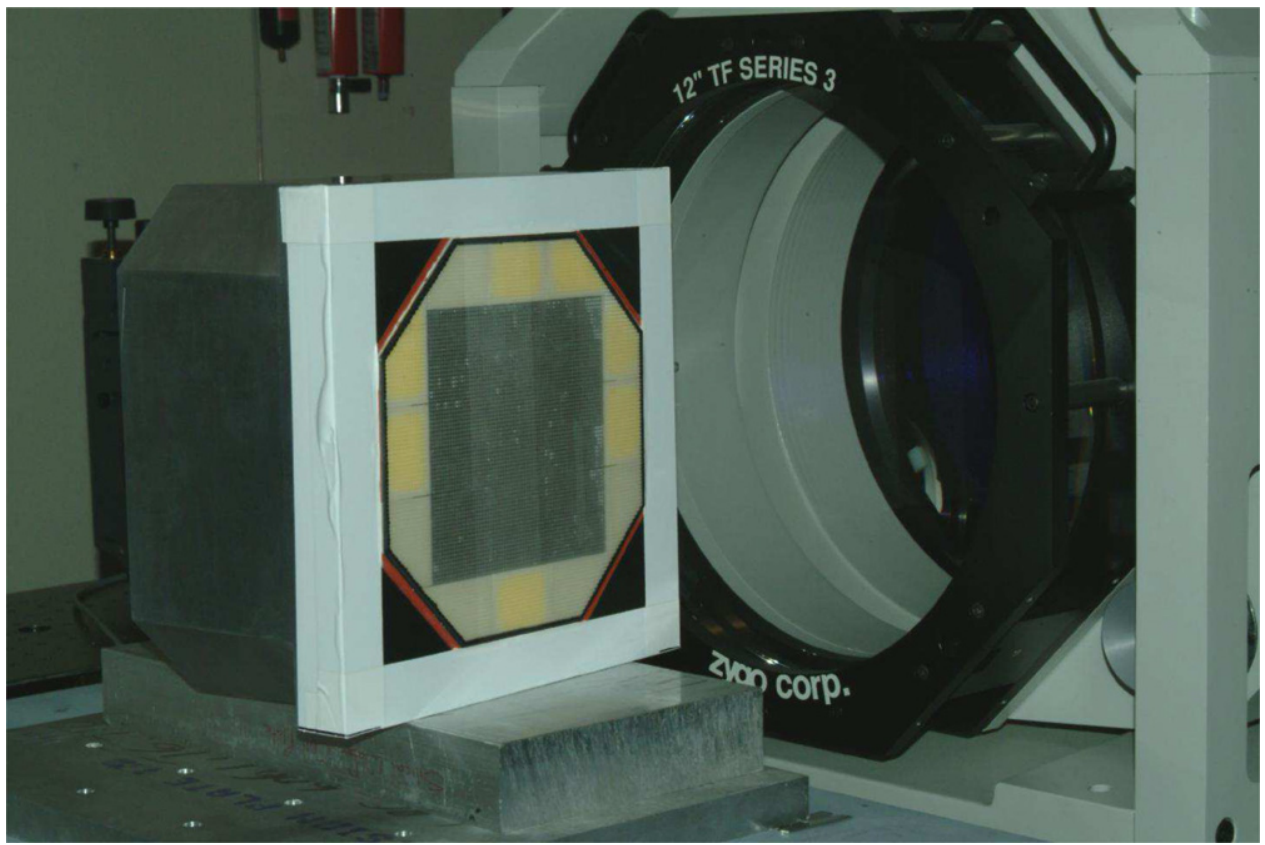

Figure 2. $66 \times 66$ actuator HODM, fabricated by Xinetics, Inc. for the PALM-3000 adaptive optics system, undergoing test during optical polishing. (A color version of this figure is available in the online journal.)

Table 1

PALM-3000 Deformable Mirrors

\begin{tabular}{lcc}
\hline \hline Parameter & Low-order Deformable Mirror (LODM) & High-order Deformable Mirror (HODM) \\
\hline Total number of actuators & 349 & 4,356 \\
Number of active actuators & 241 & 3,388 \\
Number of non-functional sctuators & 0 & 1 \\
Actuator material & Electrostrictive poled & Electrostrictive PMN \\
& Lead magnesium niobate (PMN) & \\
Construction type & Close-packed discrete actuators & 6 Mosaic of $11 \times 11$-actuator \\
$P-V$ surface stroke & & Integrated photonex modules \\
$P-V$ interactuator stroke & $4.8 \mu \mathrm{m}$ & $1.06 \mu \mathrm{m}$ \\
Available atmospheric stroke & $1 \mu \mathrm{m}$ & $0.5 \mu \mathrm{m}$ \\
Self-corrected surface figure & $\approx 3 \mu \mathrm{m}$ & $\approx 1 \mu \mathrm{m}$ \\
Actuator linearity over $80 \%$ of stroke & $19 \mathrm{~nm} \mathrm{rms}$ & $11 \mathrm{~nm}$ rms \\
Actuator pitch & $15 \%$ & $25 \%$ (bias-dependent) \\
Projected actuator pitch on-sky & $7.0 \mathrm{~mm}$ & $1.78 \mathrm{~mm}$ \\
Angle of incidence of beam & $31.25 \mathrm{~cm}$ & $8.1 \mathrm{~cm}$ \\
Facesheet type & 16.05 & 10.50 deg \\
Facesheet coating & $1.4 \mathrm{~mm} \mathrm{Zerodur}$ & $0.2 \mathrm{~mm}$ Zerodur \\
Mirror assembly mass & Protected aluminum & Protected aluminum \\
Drive electronics & $8 \mathrm{~kg}$ & $9 \mathrm{~kg}$ \\
Slaving & $0-100 \mathrm{~V} \mathrm{JPL} \mathrm{custom}$ & $0-400 \mathrm{~V}$ Xinetics-provided \\
Optical conjugate altitude & $780 \mathrm{~m}$ & None \\
Delivery date & 1998 & $0 \mathrm{~m}$ \\
Notes & Up to $15 \%$ hysteresis at $\sim 0 \mathrm{C}$ & 2010 \\
& Overdrive protected via Zener network & Stectronically slaved perimeter actuators
\end{tabular}

Summary properties of the HODM and LODM are shown in Table 1.

\subsubsection{Low-order Deformable Mirror}

The PALM-3000 LODM is the original PALM-241 DM349 (Oppenheimer et al. 1997), which has been in routine use since 1999. It was stripped and recoated on one occasion in 2003, an operation that involved removal of the mirror facesheet. In terms of operational reliability, this mirror has seen approximately 500 nights of scheduled science usage. Assuming 50\% openshutter time (year-round) and average operation at $1 \mathrm{kHz}$ update rate, this mirror has seen $\approx 7.5$ Gcycles $\times 241$ active actuators $\approx 1.8$ trillion actuator-updates in science use. We estimate that including laboratory time, $\approx 8.4$ trillion actuator-updates have been made without a single failure at the actuator level. ${ }^{5}$ This reliability data, along with similarly experiences at other observatories, is now able to inform design decisions for future generation astronomical $\mathrm{AO}$ systems.

5 During 12 yr of routine science operation, PALM-241 did on two different occasions suffer broken pins on the integrated 440 pin LODM cable connector, each resulting in the loss of function of one actuator for a period of several weeks. 


\subsubsection{High-order Deformable Mirror}

The HODM is the largest format astronomical DM to date, having $66 \times 66$ physical actuators organized into $6 \times 6$ actuator modules containing $11 \times 11$ actuators each. The actuators are made of electrostrictive lead magnesium niobate (PMN) and are spaced by $1.8 \mathrm{~mm}$ center-to-center with $0.15 \mathrm{~mm}$ gaps between the actuators. A $0.2 \mathrm{~mm}$ thick glass facesheet is attached to the actuators. The mirror has 3388 active actuators within a circular clear aperture of $117 \mathrm{~mm}$ in diameter. The AO relay produces a $114 \mathrm{~mm}$ pupil which fully illuminates 63 wavefront sensor subapertures when used in $s_{64}$ mode (spanning 64 HODM actuators in a pupil-matching Fried geometry). The entire DM housing (excluding external wiring) is $23 \times 23 \times 13.2 \mathrm{~cm}$.

The nominal $0-100 \mathrm{~V}$ range for actuation is limited by the Xinetics-provided DM drive electronics to $\pm 30 \mathrm{~V}$ about a $+40 \mathrm{~V}$ bias signal, which was found experimentally to put the HODM near the center of its linear range. The drive electronics overall require $55 \mathrm{~A}$ of continuous current, $90 \mathrm{~A}$ peak, necessitating the installation of auxiliary $120 \mathrm{~V}$ power capacity to the Cassegrain environment.

During acceptance testing, the best controlled HODM selfflattening achieved was $11 \mathrm{~nm}$ rms of residual surface error (Roberts et al. 2010). Assuming that spatial frequencies of eight cycles or less across the pupil are corrected by the LODM, then the HODM stroke required to self-flatten, in the lab, was achieved using less than 5\% rms of the available HODM stroke, with a few individual HODM actuators using up to $25 \%$ of their range.

Although the HODM represents a significant prototyping achievement for Xinetics, Inc., there were imperfections created during the manufacturing process which affect the surface figure. Because the actuators are closely spaced and made of an electrically active material, the entire grid of actuators was dipped in a conformal coating prior to attaching the facesheet. During initial polish of the completed mirror, polishing slurry leaked into the area between the actuators, causing either the conformal coating or the facesheet bonding adhesive to absorb moisture and wick between the actuators. When sent to vacuum aluminization, the offensive material dried out and was fixed in place between actuators. Thus, as the material absorbs or releases moisture, it expands and contracts. Because the actuators are rigidly attached, the inter-actuator dimensional change results in highly local deformations in the facesheet. The stroke of the majority the actuators has not been compromised, but for a group located along the bottom edge of the HODM, 18 actuators have been found to be restricted to less than half the nominal actuator stroke ( 8 of which are inscribed within our useful pupil).

A complete solution to this problem would require removal of the facesheet and an attempt to clean the unwanted material from between the actuators. In addition to risking the facesheet, this would have risked permanent damage the actuators. As an alternative mitigation, we elected to have the facesheet repolished under strict humidity control and implemented a passive relative humidity $(\mathrm{Rh})$ control system, sealing the HODM case and using a specially formulated passive, twoway $30 \% \mathrm{Rh}$ maintaining Humidipak (now Boveda) coupled to an Rh monitoring system. Results from initial winter operation indicate the HODM puckers may additionally be temperaturedependent, even with controlled $\mathrm{Rh}$, but this remains under investigation.

During early science observations, the high-spatial-frequency nature of the HODM deformations has been confirmed to move additional stellar light outside the HODM control region, beyond 1".6). Wave-optics simulations on the impact to contrast due to frequency folding effects predict residual stellar speckles to have a contrast ratio of less than $10^{-7}$.

\subsection{Wavefront Sensor}

PALM-3000 uses a Shack-Hartmann wavefront sensor with four different pupil sampling formats (Table 2) implemented through a lenslet array exchanger (Baranec 2008). The reconfigurable approach, previously adopted for the Very Large Telescope NAOS system (Rousset et al. 2003), allows performance optimization of PALM-3000 for NGSs spanning over $18 \mathrm{mag}$ of brightness (Baranec \& Dekany 2008). The Shack-Hartmann detector is a $128 \times 128$ pixel split frame transfer E2V CCD50 packaged into a SciMeasure Analytical Systems "Li'1 Joe" controller head, a platform originally developed for PALM-241 (DuVarney et al. 2000a). The camera pixels can be read out at multiple selectable rates which correspond to the full-frame readout rates, with corresponding read-noise values, presented in Table 3. To enable an effectively continuous selectable frame rate, the detector controller can be programmed with an adjustable delay time between the readout of the last pixel in the frame storage area and the execution of the frame transfer. This allows for slower frame rates than presented in Table 3 while still operating at one of the four pixel rates and read-noise values.

\subsubsection{Pupil Sampling Modes}

We index the four PALM-3000 pupil sampling modes according to the one-dimensional number of Shack-Hartmann subapertures formed across the pupil diameter, $s=\{64,32$, $16,8\} .{ }^{6}$ Each subaperture mode operates in a different diffraction regime and thus creates different subaperture PSFs. The $s_{64}$ PSFs are dominated by diffraction with large $2^{\prime \prime} .1$ pixel $^{-1}$ quad-cells, making centroiding precision particularly sensitive to CCD charge diffusion. The $s_{64}$ plate scale was chosen to give a ratio of pixel to spot size of $\approx 1$, providing reasonable linearity around the quad-cell zero-point (Hardy 1998). The plate scale for $s_{32}$ was similarly chosen for linearity if using only $2 \times 2$ pixels in NGS mode; however, when guiding on extended objects $s_{32}$ is intended to calculate a center of mass using $4 \times 4$ pixels, though full implementation of this is not yet complete. Were the pixel-to-image size ratio for $s_{8}$ mode maintained at $\approx 1$, the $f / \#$ of the microlens array would be reduced to the point where aberrations in the $\mathrm{AO}$ relay would be the dominant factor in spot size, increasing centroiding error unnecessarily. To compensate for this, we adopted the same $f / \#$ for $s_{8}$ as we selected for $s_{16}$, with the result that the $s_{8}$ plate scale is finer than that for $s_{16}$ by a factor of two.

Foreshortening of the pupil as projected onto the HODM due to the 10.5 angle of incidence by the chief ray is compensated by the wavefront sensor optics to provide true Fried geometry (Fried 1977). The optical conjugate of the LODM is at a point approximately $780 \mathrm{~m}$ above the observatory and although the system is operated in pseudo-single-conjugate mode, some astrometric degradation over the full $40^{\prime \prime} \times 40^{\prime \prime}$ PHARO field is tolerated. Although non-pupil DM imperfect motions increase the stochastic error, and thus require additional averaging to overcome noise sources, the systematic errors that arise from variations in slowly varying component of LODM shape are the most problematic for precision astrometry. In an experiment

\footnotetext{
6 For simplicity, we hereafter will refer to the physically 63 across pupil sampling mode as s64 mode.
} 
Table 2

PALM-3000 Wavefront Sensor

\begin{tabular}{|c|c|c|c|c|c|}
\hline \multirow[t]{2}{*}{ Parameter } & \multicolumn{5}{|c|}{ Wavefront Sensor Mode } \\
\hline & $s_{64}$ & $s_{32}$ & $s_{16}$ & $s_{8}$ & $s_{1}$ \\
\hline Subapertures per Pupil Dia. & 63 & 32 & 16 & 8 & 1 \\
\hline No. of majority illuminated subapertures & 2804 & 708 & 177 & 48 & 1 \\
\hline Projected subaperture diameter $(\mathrm{cm})$ & 8.06 & 15.88 & 31.75 & 63.5 & 508 \\
\hline Pixel scale on-sky $\left(\operatorname{arcsec}\right.$ pixel $\left.^{-1}\right)$ & 2.1 & 1.5 & 1.3 & 0.65 & $\cdots$ \\
\hline Subimage spacing (pixel) & 2 & 4 & 8 & 16 & $\cdots$ \\
\hline Subaperture field of view $\left({ }^{\prime \prime}\right)$ & \multicolumn{4}{|c|}{$0.48-3.84$, variable ${ }^{\mathrm{a}}$} & $\cdots$ \\
\hline Design subaperture polychromatic image FWHM (") & 2.1 & 1.5 & 1.3 & 1.3 & $\cdots$ \\
\hline Lenslet substrate & \multicolumn{4}{|c|}{$\mathrm{SiO}_{2}$} & $\cdots$ \\
\hline Lenslet pitch $(\mu \mathrm{m})$ & 150 & 300 & 600 & 1200 & $\cdots$ \\
\hline Lenslet focal length (mm) & 14.05 & 19.66 & 22.69 & 45.38 & $\infty$ \\
\hline Lenslet radius of curvature (mm) & $6.32 \% \pm 2.3 \%$ & 8.85 & 10.21 & 20.42 & $\cdots$ \\
\hline Lenslet surface sag $(\mu \mathrm{m})$ & 0.5 & 1.3 & 4.5 & 8.9 & $\cdots$ \\
\hline Lenslet focal ratio & 93.0 & 65.1 & 37.6 & 37.6 & $\cdots$ \\
\hline Nominal spatial filter setting (") & 1.92 & 0.96 & 0.48 & $\cdots$ & 3.84 \\
\hline Nominal spatial filter setting $(\mu \mathrm{m})$ & 760 & 380 & 190 & $\cdots$ & 1560 \\
\hline Subaperture pixel modes ${ }^{b}$ & $2 \times 2$ & $\begin{array}{c}c 2 \times 2 \\
4 \times 4 b 2 \times 2^{c} \\
4 \times 4\end{array}$ & $\begin{array}{c}\text { c } 2 \times 2 \\
\text { c } 4 \times 4 \text { b } 2 \times 2^{c} \\
\text { c } 4 \times 4\end{array}$ & $\begin{array}{c}\text { c } 4 \times 4 \\
\text { c } 4 \times 4 \text { b } 2 \times 2^{c} \\
\text { c } 8 \times 8 \\
\text { c } 8 \times 8 \text { b } 2 \times 2^{c}\end{array}$ & $128 \times 128$ \\
\hline WFS geometry & Fried & Non-Fried & Non-fried & Non-fried & $\cdots$ \\
\hline Notes & $\cdots$ & $\cdots$ & Low priority & $\cdots$ & Pupil img only \\
\hline
\end{tabular}

Notes.

a Set by a variable-width square field stop/spatial filter (Section 3.3.2).

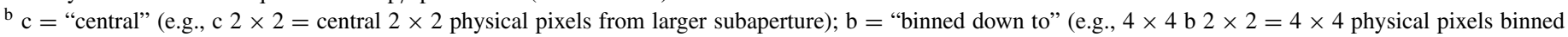
on-chip down to $2 \times 2$ pixels at readout).

${ }^{c}$ As of this writing, binning modes have yet been implemented due to emphasis to optimize high-contrast observations on bright stellar targets.

we measured the separation of a binary star pair with both a flattened LODM and after applying the full LODM stroke. Over the approximately 20 arcsec binary separation, we determined the LODM shape induced a 100 mas shift, about $0.5 \%$. Over the much smaller exoplanet working angle, and for reasonabe long-time-average LODM figure drift of $150 \mathrm{~nm} P-V$, we estimate the systematic astrometric errors from our choice of LODM conjugation should be less than $600 \mu$ as. In the long run, we expect extremely precise binary star orbits derived from Gaia data to allow us to calibrate the long-term systematic astrometric errors induced by our two-DM architecture to better than $40 \mu$.

\subsubsection{Spatial Filter}

The PALM-3000 wavefront sensor includes a variable aperture optical spatial filter (Poyneer \& Macintosh 2004; Swartzlander et al. 2008) that serves as the field stop, limiting crosstalk between sub-aperture detector pixels. It is also designed to mitigate wavefront aliasing errors that result in lower AO performance which can corrupt high-contrast observations. The spatial filter used in PALM-3000 has a square aperture ranging from 190 to $1560 \mu \mathrm{m}$, with the largest aperture corresponding to 3 ". 84 which allows the extended objects Uranus and Neptune to be used as AO guide sources. The appropriate aperture sizes for high-contrast use are shown in Table 2. We do not intend to operate the spatial filter for anti-aliasing in $s_{8}$ mode, where the contribution of aliasing to either contrast or wavefront errors is relatively small.

\subsection{Mechanics and Interfaces}

To minimize the impact on observatory operations and avoid unnecessary engineering, we reused the main optical bench, its handling cart and rotisserie fixture, and internal telescope and source simulator from PALM-241. Thus, the PALM-3000 installs horizontally at Cassegrain focus, with the AO relay optics suspended from the underside of the optical bench. Earlier finite element analyses, verified by PALM-241 flexure tests on the turnover fixture, demonstrated a total $180^{\circ}$ inversion deflection of the bench surface of approximately $1 \mathrm{~mm}$ (in this configuration, the bench is held by its endpoints within the rotisserie fixture). No appreciably local flexure of the bench face sheet due to mounted optics has been seen to date, although we take care to distribute the load from science instruments over a total face sheet area of $\approx 230 \mathrm{~cm}^{2}$. We currently limit science instruments to a volume spanning the $137 \mathrm{~cm}$ width of the AO bench $\times 45.7 \mathrm{~cm}$ depth (measured along a radius extending from the Cassegrain rotation axis) $\times 91.4 \mathrm{~cm}$ height perpendicular (and hanging below) the lower bench surface. A mass limit of $200 \mathrm{~kg}$ is set by face sheet safety margin while an axial torque limit of $34.6 \mathrm{~m} \mathrm{~kg}$ about the telescope axis is imposed to avoid back-driving of the Cassegrain ring rotation motor. The total mass installed onto the Cassegrain cage during a PALM-3000 setup with the heaviest instrument (P1640) is $\approx 3,230 \mathrm{~kg}$, which has required additional counterweights to be added to the top of the telescope ring for balance. Despite the large weight, no detriment to any telescope performance parameter has been noted. 
Table 3

PALM-3000 Wavefront Sensor Detector

\begin{tabular}{|c|c|c|}
\hline & Value \\
\hline \multicolumn{2}{|l|}{$\frac{\text { Parameter }}{\text { Detector type }}$} & E2V CCD50 \\
\hline \multirow{3}{*}{\multicolumn{2}{|c|}{$\begin{array}{l}\text { Pixel size } \\
\text { Camera manufacturer } \\
\text { Dark current ( } 278 \mathrm{~K} \text { ambient) }\end{array}$}} & $24 \mu \mathrm{m}$ \\
\hline & & SciMeasure Analytical Systems, Inc \\
\hline & & $150-780 \mathrm{e}^{-}$pixel $^{-1} \mathrm{~s}^{-1}$ \\
\hline Readout rate & Readout noise & Camera gain \\
\hline $2000 \mathrm{fps}$ & $9.3 e-$ & $0.1 e-/ \mathrm{DN}$ \\
\hline $1200 \mathrm{fps}$ & $7.3 e-$ & $0.4 e-/ \mathrm{DN}$ \\
\hline $500 \mathrm{fps}$ & $3.9 e-$ & $0.7 e-/ \mathrm{DN}$ \\
\hline $200 \mathrm{fps}$ & $3.1 e-$ & $2.5 e-/ \mathrm{DN}$ \\
\hline
\end{tabular}

\subsection{Real-time Computation}

\subsubsection{Reconstructor Pipeline and Wavefront Control}

The large number of DM actuators in the PALM-3000 system requires an accurate reconstructor matrix, mapping centroid measurements to wavefront phase, in order to achieve robust closed-loop stability (Brennan \& Kim 2001). The system experiences significant pupil shifts in response to changes in telescope declination, thus the reconstructor must be periodically re-identified throughout the course of an observing period. To facilitate this process, PALM-3000 possesses a unified reconstructor pipeline that autonomously gathers current telescope data and computes a new reconstructor matrix.

The telescope operator initiates the computation by calling the reconstructor pipeline script. The script is structured such that pertinent reconstructor parameters can be specified if desired, however the default parameters are typically sufficient and no further input from the user is required. The pipeline begins by gathering and processing basic observing information, such as telescope position and subaperture flux measurements. The reconstructor matrix itself is the result of a regularized and weighted pseudo-inverse performed on precomputed actuator influence matrices. The weighting and regularization terms incorporate pupil illumination information, as well as a priori Kolmogorov phase covariance values that may be adjusted based on the prevailing seeing (Law \& Lane 1996). The inversion calculation is computationally intensive, thus the HODM and LODM reconstructor computations may be performed together or separately on remote servers or CPU cores. Once the HODM and LODM reconstructors are computed, they are combined into a single matrix and automatically loaded directly into the PALM-3000 servo controller. Two additional rows are added to account for tip/tilt residuals. An optional "offload" matrix is also computed, which projects HODM actuator positions to the space of LODM actuators.

During each control loop iteration, the PALM-3000 real-time computer (RTC) multiplies centroid measurements with the reconstructor matrix to provide a residual phase estimate in DM actuator space. The wavefront controller consists of parallel "leaky" integrators for the HODM, LODM and fast-steering mirror, which act on the residuals to generate position commands. The fast-steering mirror alone does not provide the necessary bandwidth to fully compensate for tilt/tip errors, thus a portion of these residuals are offloaded to the LODM. To harness the large stroke range of the LODM, an alternative control scheme offloads HODM residuals to the LODM integrator, effectively creating a double-integral controller for low order aberrations. Control gains can be adjusted during closed-loop operation to optimize system performance and maintain stability.

\subsubsection{Real-time Computer}

The PALM-3000 RTC for wavefront reconstruction is based on a GPU implementation of 16 retail NVIDIA 8800 GTX graphics cards distributed over eight dual-core Opteron PCs from Hewlett-Packard, each hosting two cards (Truong et al. 2008) in conjunction with a Servo PC that combines partial reconstruction results and a Database PC which provides high-speed telemetry. The GPU architecture provides supercomputing-like power and memory bandwidth coupled with ease of programming through a low-level C interface. All 10 PCs are interconnected using a Quadrics QsNetII 16 port switch that delivers over $900 \mathrm{MB} \mathrm{s}^{-1}$ of user space to user space bandwidth each direction with latency under $2 \mu$ as for a total of $14.4 \mathrm{~GB} \mathrm{~s}^{-1}$ of bisectional bandwidth and broadcast capability. High-bandwidth AO telemetry data, such as wavefront sensor pixel data, as well as latency-sensitive data such as HODM commands, are transferred using fiber optics. Low-bandwidth data, such as acquisition camera pixels and electronics hardware status, are sent from the Servo PC to the Database PC via a dedicated $1 \mathrm{Gbit}$ Ethernet. Long latency-tolerant commands, such as those issued by the Servo PC to stepper motors and lamps are sent via direct connections.

The measured mean processing latency of the wavefront reconstruction based on a full matrix vector multiplication using the input matrix of 4096 actuator values by 8192 centroid values and $2 \times 2$ pixel centroiding has been measured to consist of the following terms: $94 \mu$ s for centroiding and vectormatrix-multiply in parallel on eight PCs, $41 \mu \mathrm{s}$ transfer of partial residuals to combining PC, $70 \mu$ s for servo calculation setting the new actuator commands, $35 \mu$ s for software interactuator voltage check to protect the HODM from potentially damaging strain (reducible to $10 \mu \mathrm{s}$ via faster algorithm), $125 \mu \mathrm{s}$ communication through the DM interface board (reducible to $25 \mu$ s by reimplementing the Curtiss-Wright driver ourselves), and $50 \mu$ s inter-actuator voltage check in FPGA hardware. Startof-data to end-of-data delay was confirmed at $475 \mu \mathrm{s} \pm 25 \mu \mathrm{s}$ jitter. In addition $30 \mu$ s of frame transfer delay in the wavefront sensor controller. Latency is measured from the falling edge of Start of Frame signal (e.g., the end of frame transition) on the Little Joe Camera transmitting CameraLink card, to the 50\% transition point of an actuator's drive voltage and does not include the camera integration time. Measurement via oscilloscope has shown this latency to be $956 \mu$ as for $1250-2000 \mathrm{~Hz}$ frame rate readout and $1280 \mu$ as for camera readout in the range of 300-1249 Hz. The mean compute latency in the real-time controller has been benchmarked at $415 \mu \mathrm{as}$, with standard deviation of $30 \mu$ as.

The HODM driver is specified for a $3 \mathrm{~A}$ current limit. The rise time of a single actuator, settling to within $5 \%$ of a $300 \mathrm{~nm}$ step 
Table 4

PALM-3000 Commissioned Instrument Suite

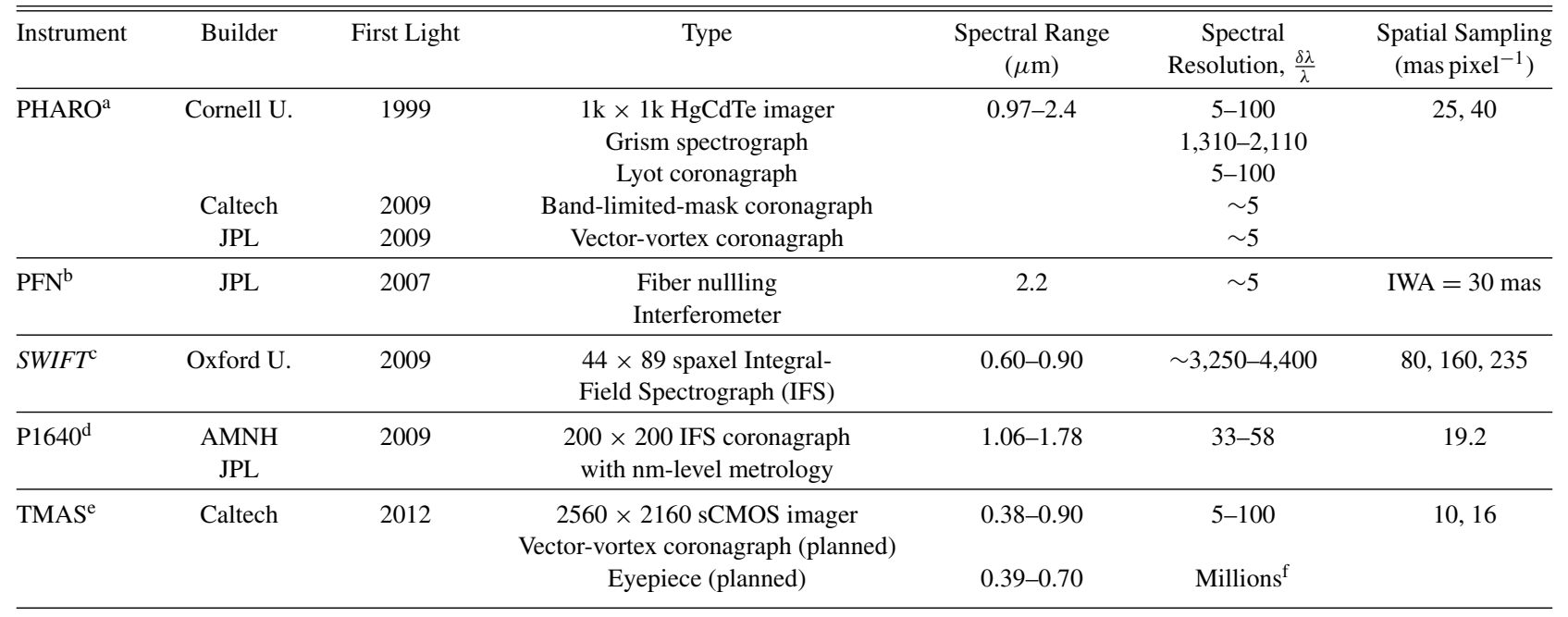

Notes.

${ }^{a}$ Hayward et al. (2001).

b Hanot et al. (2011).

c Thatte et al. (2010).

d Hinkley et al. (2011).

e R. Dekany et al. (2013, private communication).

${ }^{\mathrm{f}}$ Geldard (1972).

function, was measured to be $20 \mu \mathrm{s}$, consistent with this current limit. The HODM driver latency, measured from the sending of a digital command to the setting of the actuator voltage, was found to be $60 \mu \mathrm{s}$. Both of these measurements confirm the design specifications of our drive electronics.

\subsubsection{Wavefront Calibration}

Non-common-path static wavefront calibration for PALM3000 is implemented via an interface allowing for the loading of element slope offset vectors in the appropriate pupil mode which represents the target command point in the real-time AO wavefront reconstruction. These slope offsets, represented internally in Shack-Hartmann sensor pixel coordinates, are generated via different techniques depending on the science camera undergoing calibration. For PHARO, we use a modified iterative Gerscherg-Saxton phase diversity algorithm to achieve wavefront calibration to better than $40 \mathrm{~nm}$ rms phase error (Roberts et al. 2012; Burruss et al. 2010). For P1640, an electric field measuring calibration interferometer has so far achieved approximately $14 \mathrm{~nm}$ wavefront error (G. Vasisht 2013, private communication).

\subsection{Cooling Infrastructure}

Prior to PALM-3000 development, the Hale Telescope at Palomar had no capacity to provide instrumentation with facility liquid cooling. Experimental investigation of the PALM-241 system, which dissipated a total of $1.5 \mathrm{~kW}$ below the primary mirror determined this heat flow led to an approximately $1{ }^{\circ} \mathrm{C}$ temperature rise in the area of the primary mirror directly above the dissipating electronics. While not directly detrimental to PALM-241 observations, the thermal inertia of the primary mirror demonstrated residual surface distortions for up to $40 \mathrm{hr}$, degrading some non-AO operations, particularly after comprehensive refurbishment of the Hale primary mirror back supports improved seeing-limited image quality in 2009.
As the PALM-3000 AO system dissipates approximately 7 $\mathrm{kW}$ during HODM operation, the project and Palomar staff jointly undertook the addition of a liquid cooling system to remove heat from the electronics in the Cassegrain cage area. The new cooling system comprises a primary facility chiller in conjunction with a secondary process chiller, which circulates coolant to multiple fan tray heat exchangers operating within each rack. The facility chiller ejects its heat through an exhaust tunnel which is vented a sufficient distance from the telescope dome. The diluted glycol coolant is currently conveyed to the Cassegrain cage by way of a draped umbilical cord, which also provides additional electrical power and fiber data lines required for PALM-3000. Despite the additional torque of the draped umbilical, no noticeable change to the telescope pointing performance has been found after a routine pointing model recalibration.

\section{INSTRUMENT SUITE}

PALM-3000 has thus far been commissioned with five science instruments as summarized in Table 4. The range of science capability provided by the diverse PALM-3000 instrument suite includes direct near-infrared and visible imaging, slit-based near-infrared grism spectroscopy, moderate visible resolution and low near-infrared resolution integral field spectroscopy, coronagraphy, and near-infrared nulling interferometry. Fastframe region-of-interest array imaging is also available in both the visible and near-infrared.

\section{PREDICTED PERFORMANCE}

\subsection{Error Budgets}

PALM-3000 is capable of minimizing the residual wavefront error for guide stars that range in brightness by over a factor of $10^{7}$ through adjusting the combination of wavefront sensor pupil sampling, camera frame rate, and servo control modes. Naturally, the delivered level of wavefront correction over this broad range of guide star brightness varies significantly 
Table 5

PALM-3000 Predicted Performance Error Budget

\begin{tabular}{|c|c|c|}
\hline rms Error Term & $\begin{array}{c}\text { High-contrast Exoplanet Imaging } \\
V=7 \\
\text { s64 Mode }\end{array}$ & $\begin{array}{c}\text { Kepler Candidate Follow-up } \\
V=16 \\
\text { s8 Mode }\end{array}$ \\
\hline Atmospheric fitting error ${ }^{\mathrm{a}}(\mathrm{nm})$ & 44 & 213 \\
\hline Bandwidth error $^{\mathrm{b}}(\mathrm{nm})$ & 46 & 220 \\
\hline Measurement error $^{\mathrm{c}}(\mathrm{nm})$ & 41 & 202 \\
\hline Static calibration error $(\mathrm{nm})$ & 30 & 30 \\
\hline Other high-order errors ${ }^{\mathrm{d}, \mathrm{e}}(\mathrm{nm})$ & 65 & 103 \\
\hline Total high-order wavefront error (nm) & 105 & 382 \\
\hline Tip-tilt errors ${ }^{\mathrm{f}}$ (mas) & 2.8 & 22 \\
\hline$V$ & 0.32 & $\ldots$ \\
\hline$I$ & 0.58 & $\cdots$ \\
\hline \multicolumn{3}{|l|}{ Predicted strehl ratio } \\
\hline$H$ & 0.88 & 0.08 \\
\hline$K$ & 0.93 & 0.23 \\
\hline
\end{tabular}

Notes.

${ }^{\text {a }}$ Atmospheric $r_{0}(500 \mathrm{~nm})=9.2 \mathrm{~cm}$ at $\zeta=10^{\circ}$ zenith angle; $\alpha_{D M}=.28$.

b Atmospheric $\tau_{0}(500 \mathrm{~nm})=3.0 \mathrm{~ms}$; bright star $2 \mathrm{kHz}$ frame rate, $-3 \mathrm{db}$ bandwidth $=100 \mathrm{~Hz}$; faint star $182 \mathrm{~Hz}$ frame rate, $-3 \mathrm{db}$ bandwidth $=12 \mathrm{~Hz}$

${ }^{\mathrm{c}}$ Total WFS photodetection efficiency $=0.24$.

${ }^{\mathrm{d}}$ Includes uncorrectable instrument errors, multispectral error, scintillation error, wavefront sensor scintillation error, DM finite stroke and digitization errors, and imperfect anti-aliasing residual error (Hardy 1998); does not include angular anisoplanatism.

${ }^{\mathrm{e}}$ Includes tip-tilt measurement, bandwidth, centroid anisoplanatism, chromatic dispersion, vibration, and non-commonpath mechanical drift errors; no angular anisokineticism.

${ }^{\mathrm{f}}$ Bright star $-3 \mathrm{db}$ tip-tilt bandwidth $=35 \mathrm{~Hz}$; faint star $-3 \mathrm{db}$ tip-tilt bandwidth $=10 \mathrm{~Hz}$.

(Table 5). Insight into the intrinsic wavefront error arising from input guide star photon noise, temporal delay due to finite integration time, and finite spatial correction bandwidth as a function of theoretical continuous pupil sampling density, $s$ (samples per pupil diameter), is shown in Figure 3.

PALM-3000 has adopted a strategy of performing the highest bandwidth tip-tilt correction using the relatively limited stroke of the LODM, coupled to a moderate-speed offload through the servo control law to the independent, large-stroke tiptilt mirror. The $-3 \mathrm{db}$ tip-tilt rejection bandwidth of the tip-tilt mirror direct loop has been measured to be $18 \mathrm{~Hz}$, while that of the cascaded approach is expected upon full implementation to achieve $>40 \mathrm{~Hz}$. Because the typical fast stroke usage of the LODM for tip-tilt correction is only a few hundred nanometers rms, there is generally little impact to the available stroke for higher-mode corrections, though the impact of spurious tilt events to LODM stability remains under investigation.

\subsection{Performance versus Guide Star Magnitude}

As with all NGS AO systems, PALM-3000 correction performance is a function of the guide star brightness. Unlike many other systems, however, the variable Shack-Hartmann pupil sampling of PALM-3000, combined with the array of selectable wavefront sensor camera frame rates, allows for quasi-continuous optimization of performance. The predicted performance for the optimal choice of pupil and camera setting is shown in Figure 3.

\section{INITIAL RESULTS}

On the nights of 2011 June 20-22, the combination of LODM and HODM correction was initially applied to a stellar source. In visible $(\lambda=0.55 \mu \mathrm{m})$ seeing of $0^{\prime} .9$, PALM-3000 achieved a first-light $K$-band Strehl Ratio of $79 \%$ as measured by $30 \mathrm{~s}$ exposures in the PHARO imaging camera, exceeding the highest Strehl ever obtained with PALM-241. This corresponded to an effective wavefront error of $170 \mathrm{~nm}$ rms. Several recognized limitations were known at the time, such as our having only partially implemented our reconstructor pipeline and not yet fully implemented the MGS calibration algorithm with PHARO. During the initial 12 months following our PALM-3000 first lock, the AO team was engaged in commissioning back-end science instruments at an average rate of one every three months, limiting the engineering effort available for AO performance optimization.

Since 2012 June, PALM-3000 performance has steadily improved through detailed analyses referencing against the design error budget. This process revealed an unexpected chromatic aberration, lateral color, in the high-order wavefront sensor optical relay. This chromatic magnification error, which varies with guide star effective temperature, induced an effective achromatic focus error in the science beam equivalent to up to $80 \mathrm{~nm} \mathrm{rms} \mathrm{wavefront} \mathrm{error} \mathrm{if} \mathrm{uncompensated.} \mathrm{We} \mathrm{have}$ subsequently corrected this aberration with a minor change to the high-order wavefront sensor relay optics. Similarly, small flexure-based pupil illumination changes were discovered to impact performance as a function of zenith pointing. In response, our team has implemented automations to build new reconstruction matrices based on the measured pupil illumination function, pointing anywhere on the sky.

\subsection{Current Performance of $s_{64}$ Pupil Sampling Mode}

Ongoing system optimization has thus far achieved residual wavefront error as low as $141 \mathrm{~nm}$ rms on bright guide stars. This was achieved on UT 2012 September 26 in $\mathrm{s}_{64}$ mode when guiding on the $V=3.41$ star, SAO 074996, and observing near zenith in approximately 1.0 arc sec FWHM 


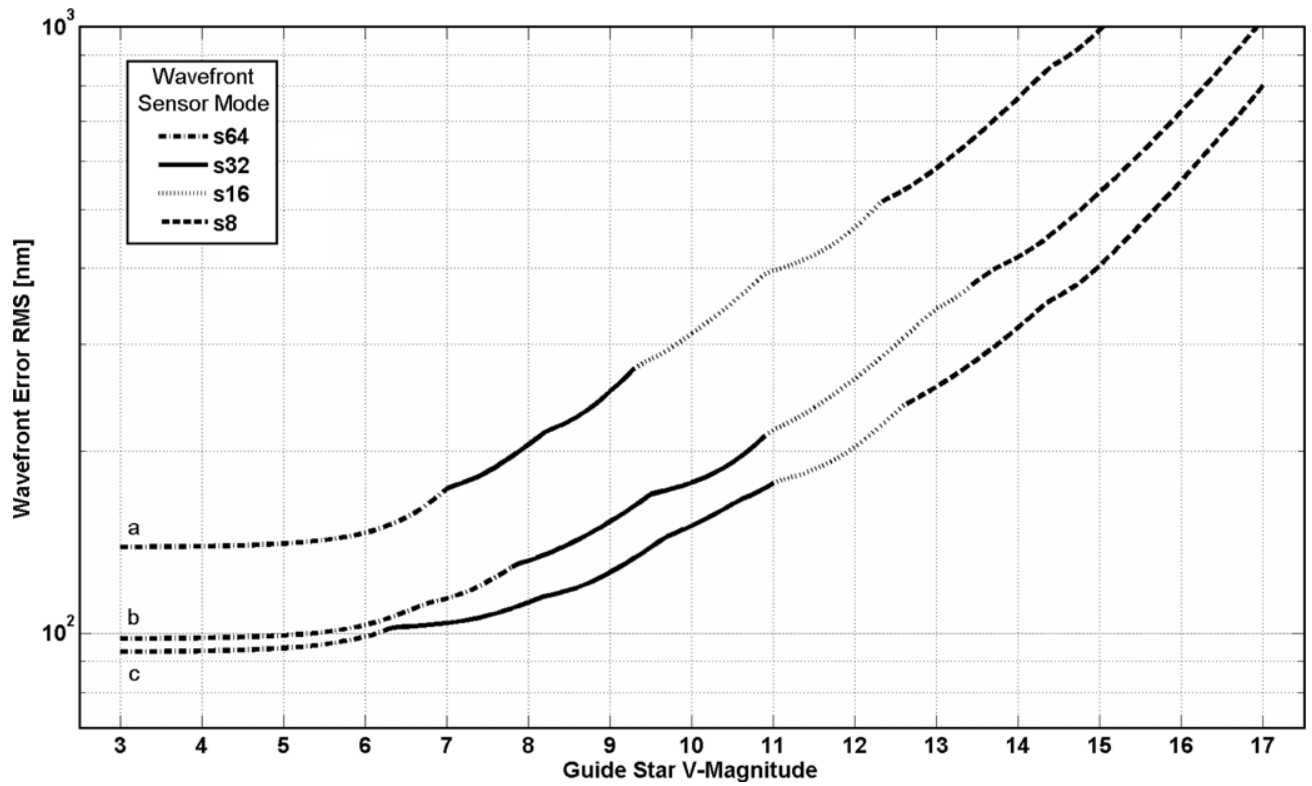

Figure 3. Performance prediction for PALM-3000, with appropriate choice of optimal wavefront sensor mode and high-order wavefront sensor frame rate, under assumed conditions, (a) $r_{0}=6.0 \mathrm{~cm}, r_{0_{\text {eff }}}=5.5 \mathrm{~cm}$ at $t_{0_{\mathrm{eff}}}=0.79 \mathrm{~ms} ;$ (b) $r_{0}=9.2 \mathrm{~cm}, r_{0_{\mathrm{eff}}}=8.4 \mathrm{~cm}, t_{0_{\mathrm{eff}}}=2.46 \mathrm{~ms} ;$ and (c) $r_{0}=15 \mathrm{~cm}, r_{0_{\mathrm{eff}}}=13.8 \mathrm{~cm}, t_{0_{\mathrm{eff}}}=$ $2.46 \mathrm{~ms}$, where the atmospheric parameters at zenith have been scaled to effective parameters corresponding to $30^{\circ}$ zenith angle.

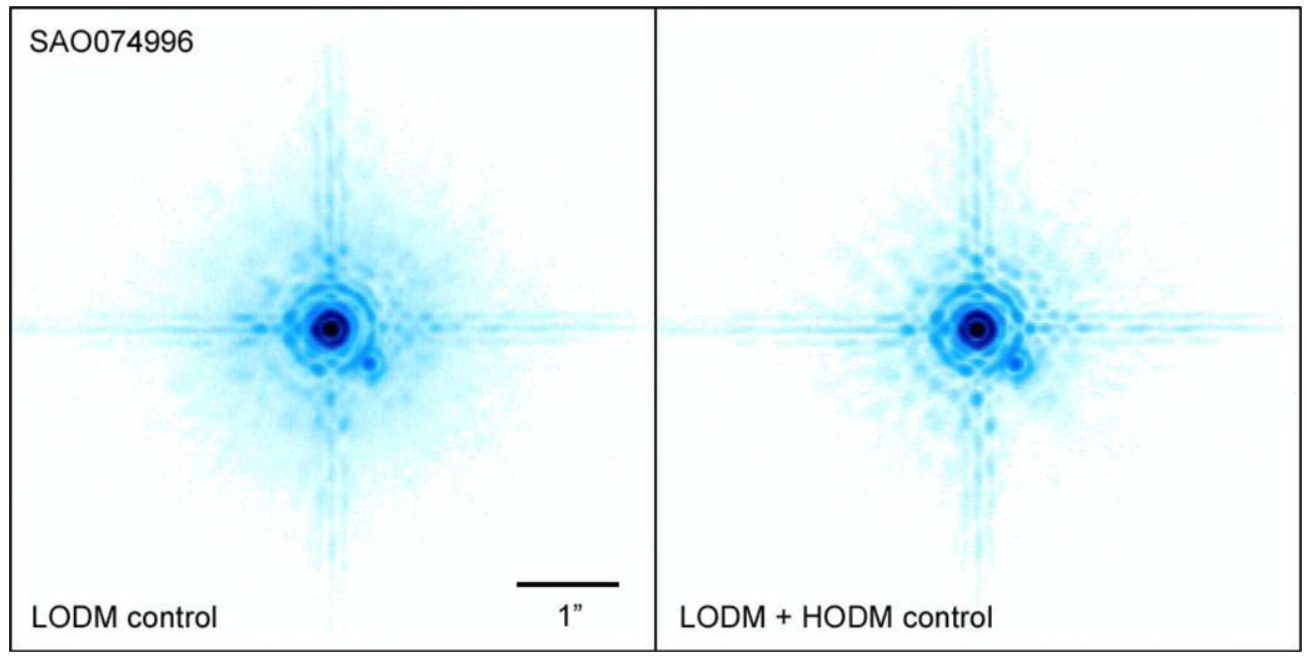

Figure 4. Logarithmic stretch infrared long-exposure observations of SAO074996 controlling only the LODM (left) and the combination of the LODM and HODM (right). Under LODM control, the working angle for clearing out atmospheric speckles is merely $4 \lambda / \mathrm{D}$. With additional HODM control, the working angle opens to $32 \lambda / \mathrm{D}$, significantly clearing away the atmospheric halo that underlies the diffraction-induced point-spread function. Note, a ghost reflection, from filters in the PHARO imager, appears to the lower right of the star in these images.

(A color version of this figure is available in the online journal.)

seeing. Figure 4 shows a long-exposure image (logarithmic stretch) of this target in the $K_{s}$ filter, under two operating configurations: LODM correction only and combined LODM+HODM correction. Each image consists of a series of PHARO images forming total integration of 272 s. For the LODM-correction-only image, the HODM was set to a fixed optimal figure previously determined using internal calibration sources. The $K_{s}$ Strehl ratio is measured using our aforementioned technique to be $72 \%$. This is somewhat underperforming our error budget prediction for LODM-only operation. The second image was formed running PALM3000 with concurrent LODM and HODM correction, delivering $K_{s}$ Strehl ratio of $85 \%$. The equivalent wavefront error of $141 \mathrm{~nm} \mathrm{rms} \mathrm{still} \mathrm{falls} \mathrm{short} \mathrm{of} \mathrm{the} \mathrm{design} \mathrm{requirement}$ of $105 \mathrm{~nm}$ rms on bright stars. We continue to optimize our woofer/tweeter control law and verify parameters following our error budgets.

In terms of clearing out the PSF within the IWA, the striking advantage of the HODM control is obvious. Any coronagraph optimized to reduce image diffraction, particularly in the range between the outer working angle of the $\operatorname{LODM}(4 \lambda / \mathrm{D})$ and that of the HODM $(32 \lambda / D)$, will enjoy the strong benefit of PALM3000 clearing away residual energy from the seeing halo. Note, conditions were exceptionally stable on the night of this test and we have no reason to believe conditions varied appreciably in the five minutes that elapsed between these two measurements.

Initial investigations of PALM-3000 performance versus guide star brightness have been limited to the initially commissioned $s_{64}$ mode, as shown in Figure 5 below. Between our initial tests in 2011 August and subsequent observations in 2012 


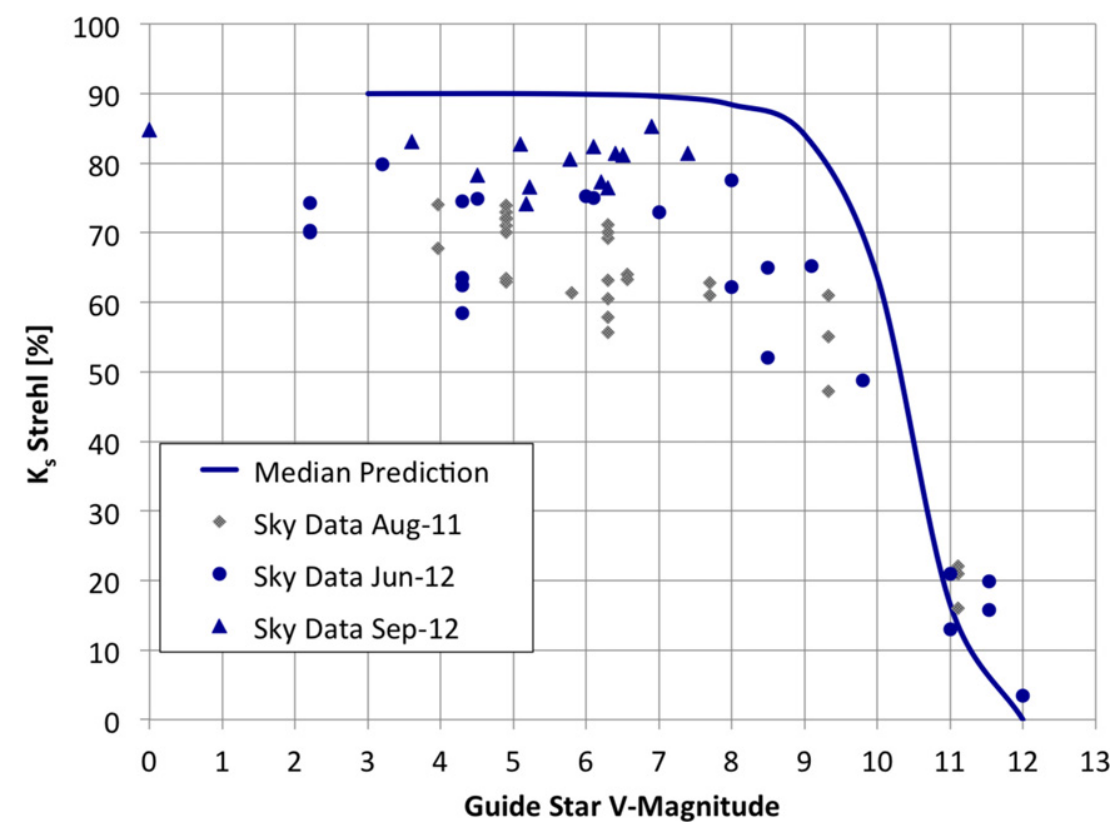

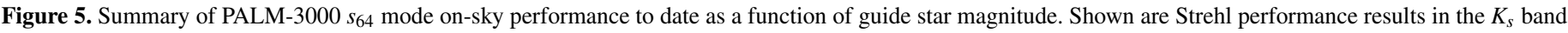

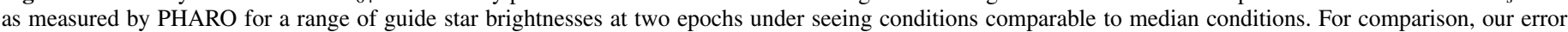

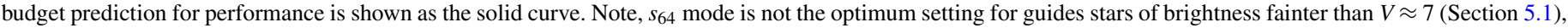
but engineering demonstrations to $V \approx 13$ to date have validated the faint star performance of the wavefront sensor camera.

(A color version of this figure is available in the online journal.)

September, a number of the initial limitations to performance have been addressed. However, performance has not yet fully reached the error budget expectation and system optimization continues.

\subsection{Planetary Science Capability}

The diffraction limited resolution of the $5.1 \mathrm{~m}$ Hale Telescope is 16 mas at $0.4 \mu \mathrm{m}$ wavelength, comparable to the planned Thirty Meter Telescope resolution in $K$ band (Dekany et al. 2003). Although we expect PALM-3000 to achieve low bluewavelength Strehl ratio performance in all but the most favorable seeing conditions, significant improvement can be obtained from short-exposure images using post-processing techniques developed at Palomar (Law et al. 2009) and elsewhere (Asensio Ramos \& López Ariste 2010; Schödel et al. 2013). Observations of solar system objects at these high angular resolutions, particularly with SWIFT (Tecza et al. 2012; Thatte et al. 2010), are already underway.

An example of this potential is shown in Figure 6, where $1000.5 \mathrm{~s}$ exposure images taken on 2012 September 27 of Ganymede taken in each of $B, R$, and $I$ filters have been combined, without frame selection, into a false-color visual image following wavelet high-pass filter transformation. In particular, solar system bodies subject to resurfacing, such as Io, Titan, and Triton, may in the future be periodically monitored at this resolution using PALM-3000.

\subsection{Future Work}

We have reported upon the initial on-sky demonstration of the $s_{64}$ subaperture correction mode of PALM-3000. Performance optimization in this mode to achieve wavefront errors as low as $\sigma=105 \mathrm{~nm}$ rms in median seeing are currently underway. Additional work will be required to implement the $s_{32}$ and $s_{8}$ pupil sampling modes, while implementation of $s_{16}$ operation has been indefinitely deferred by funding limitations. Support of routine science operations has been fully transferred to Palomar Observatory staff, though engineering optimization for performance and observing efficiency continues through successive releases of additional $\mathrm{AO}$ system automation and calibration software.

To date, all published PALM-3000 results have been based on the strategy of phase conjugation, which attempts to minimize the delivered science wavefront phase error. Optimized performance for exoplanet contrast, however, will require implementation of full electric field (phase and amplitude) correction. We have implemented both speckle nulling (Bordé \& Traub 2006) and Electric Field Conjugation (Give'on et al. 2007) algorithms for laboratory testing. On-sky demonstration of these techniques with PALM-3000 remains a topic for future publication.

\section{CONCLUSIONS}

PALM-3000 is the key capability at the heart of a multidimensional science emphasis on exoplanet discovery and analysis. Used in combination with the P1640 specklesuppression coronagraph, PALM-3000 is the first realized AO system designed to achieve $10^{-7}$ contrast direct imaging and low-resolution near-infrared spectroscopy of exoplanets using a multi-layered approach of high-order AO, a spatially filtered Shack-Hartmann wavefront sensor, an apodized-pupil Lyot coronagraph, a speckle suppression IFS, and nm-level accuracy calibration interferometer producing a dark hole in the stellar speckle field on sky. Our northern survey of A- and F-type stars in now underway. When combined with small IWA = 0!'1 coronagraphs within PHARO and TMAS, PALM-3000 will also probe star-forming associations at distances of $150-300 \mathrm{pc}$ at more modest, yet still unprecedented contrast levels. With outer working angle as large a 3".2, PALM-3000 will also probe a unique long-period orbital parameter space for exoplanets around nearby stars, including long-duration radial-velocity trend stars identified at Keck Observatory and elsewhere. 

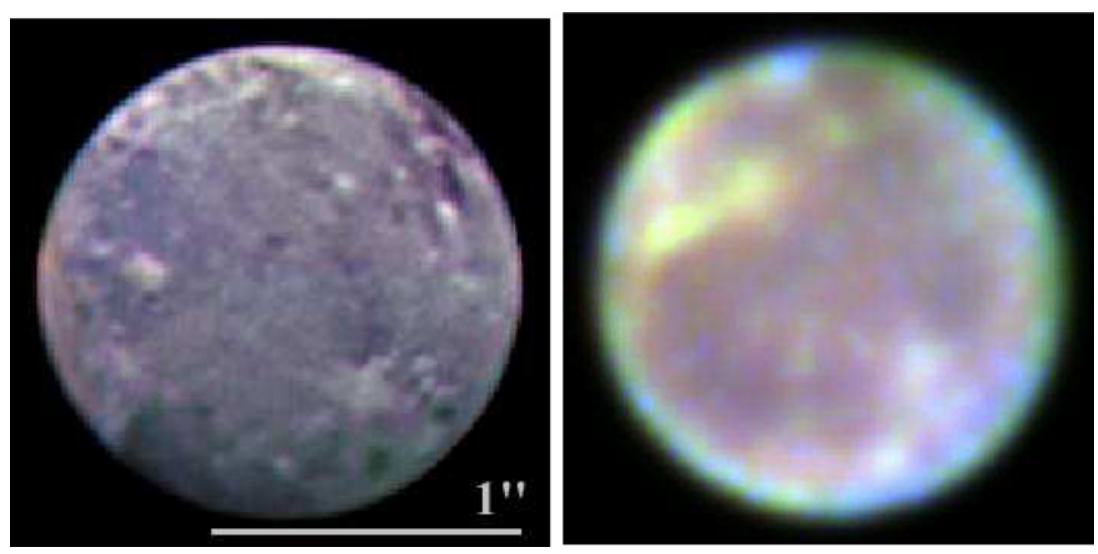

Figure 6. Left: Johnson-Cousins BRI false-color image of Ganymede, obtained with PALM-3000 and TMAS, demonstrating the visible-light correctional capability of the AO system. The pixel sampling in this image is a mere 10 mas, corresponding to about $35 \mathrm{~km}$ at the distance of Ganymede. Right: Hubble Space Telescope false-color image of Ganymede for comparison (NASA image).

(A color version of this figure is available in the online journal.)

As the highest actuator count AO system built to date for astronomical or, to our knowledge, any other purpose PALM-3000 is also being used for visible-light science at spatial resolutions as fine as 16 mas for studies of several solar system bodies and circumstellar material around bright nearby stars. Finally, PALM-3000 is successfully demonstrating technical solutions at the scale of DM and RTC capability required for the coming generation of 25-40 m diameter telescopes.

This work was performed with financial support of National Science Foundation (NSF) through awards AST-0619922 and AST-1007046, Jet Propulsion Laboratory, Caltech Optical Observatories, and the generous philanthropy of Ron and Glo Helin. Development of the Xinetics, Inc. The PALM-3000 HODM was funded by NASA SBIR award \#NNG05CA21C. The successful deployment of PALM-3000 could not have been possible without the notable talent and dedication of the entire Palomar Observatory staff. We gratefully acknowledge the specific contributions from Dan McKenna, John Henning, Steve Kunsman, Kajsa Peffer, Jean Mueller, Kevin Rykowski, Carolyn Heffner, Steve Einer, Greg van Idsinga, Mike Doyle, and Bruce Baker in interfacing to the Hale Telescope and to obtaining the commissioning data described herein.

Facilities: Hale (PALM-3000), Hale (P1640), Hale (TMAS), Hale (PHARO)

\section{REFERENCES}

Angel, J. R. P. 1994, Natur, 368, 203

Asensio Ramos, A., \& López Ariste, A. 2010, A\&A, 518, A6

Baranec, C. 2008, Proc. SPIE, 7015, 70155M

Baranec, C., \& Dekany, R. 2008, ApOpt, 47, 5155

Beichman, C. A., Krist, J., Trauger, J. T., et al. 2010, PASP, 122, 162

Bernat, D., Bouchez, A. H., Ireland, M., et al. 2010, ApJ, 715, 724

Bloemhof, E. E., Dekany, R. G., Troy, M., \& Oppenheimer, B. R. 2001, ApJL, 558, L71

Bloemhof, E. E., Marsh, K. A., Dekany, R. G., et al. 2000a, Proc. SPIE, 4007, 889

Bloemhof, E. E., Oppenheimer, B. R., Dekany, R. G., et al. 2000b, Proc. SPIE, 4007, 839

Boccaletti, A., Chauvin, G., Lagrange, A.-M., \& Marchis, F. 2003, A\&A, 410,283

Bordé, P. J., \& Traub, W. A. 2006, ApJ, 638, 488

Bouchez, A. H., Dekany, R. G., Angione, J., et al. 2007, BAAS, 38, 834

Brennan, M. J., \& Kim, S.-M. 2001, JSV, 246, 281

Burruss, R. S., Serabyn, E., Mawet, D. P., et al. 2010, Proc. SPIE, 7736, 77365X

Chauvin, G., Lagrange, A.-M., Beust, H., et al. 2012, A\&A, 542, A41
Chiang, E., Kite, E., Kalas, P., Graham, J. R., \& Clampin, M. 2009, ApJ, 693,734

Crepp, J. R., \& Johnson, J. A. 2011, ApJ, 733, 126

Davies, R., \& Kasper, M. 2012, ARA\&A, 50, 305

Dekany, R., Bouchez, A., Britton, M., et al. 2006, Proc. SPIE, 6272, 62720

Dekany, R., \& Holm, R. 1989, Conic and Nested Cone Retroreflectors, $18005 / 7502$

Dekany, R. G., Bauman, B. J., Gavel, D. T., et al. 2003, Proc. SPIE, 4839, 1165

Dekany, R. G., Wallace, J. K., Brack, G., Oppenheimer, B. R., \& Palmer, D. 1997, Proc. SPIE, 3126, 269

DuVarney, R., Bleau, C., Motter, G., et al. 2000a, in Proc. 4th ESO CCD Workshop, ed. P. Amico \& J. W. Beletic (Astrophysics and Space Science Library, Vol. 252; Dordrecht: Kluwer), 395

DuVarney, R., Bleau, C., Motter, G., et al. 2001, ExA, 11, 237

DuVarney, R. C., Bleau, C. A., Motter, G. T., et al. 2000b, Proc. SPIE, 4007, 481

Femenía Castellá, B., Labadie, L., \& Rebolo López, R. 2010, Proc. SPIE, 7736 77363

Fried, D. L. 1977, JOSA, 67, 370

Garrel, V., Guyon, O., Baudoz, P., et al. 2011, Proc. SPIE, 8151, 81510R

Geldard, F. A. 1972, The Human Senses (2nd ed.; New York: John Wiley \& Sons)

Give'on, A., Kern, B., Shaklan, S., Moody, D. C., \& Pueyo, L. 2007, Proc. SPIE, 6691,66910

González-García, B. M., Zapatero Osorio, M. R., Béjar, V. J. S., et al. 2006, A\&A, 460, 799

Haisch, K. E., Jr., Lada, E. A., \& Lada, C. J. 2001, ApJL, 553, L153

Hanot, C., Mennesson, B., Martin, S., et al. 2011, ApJ, 729, 110

Hardy, J. W. 1998, Adaptive Optics for Astronomical Telescopes (New York: Oxford Univ. Press)

Hart, M. 2010, ApOpt, 49, D17

Haubois, X., Perrin, G., Lacour, S., et al. 2009, A\&A, 508, 923

Hayward, T. L., Brandl, B., Pirger, B., et al. 2001, PASP, 113, 105

Hinkley, S., Oppenheimer, B. R., Zimmerman, N., et al. 2011, PASP, 123, 74

Johnson, J. A., Butler, R. P., Marcy, G. W., et al. 2007, ApJ, 670, 833

Jolissaint, L., Véran, J.-P., \& Conan, R. 2006, JOSAA, 23, 382

Kalas, P., Graham, J. R., Chiang, E., et al. 2008, Sci, 322, 1345

Kibblewhite, E. 2008, Proc. SPIE, 7015, 70150M

Kibblewhite, E. 2009, in Proc. Advanced Maui Optical and Space Surveillance Technologies Conference, ed. S. Ryan (Maui, HI: Maui Economic Development Board), E33

Kraus, A. L., Ireland, M. J., Martinache, F., \& Lloyd, J. P. 2008, ApJ, 679, 762 Lagadec, E., Verhoelst, T., Mékarnia, D., et al. 2011, MNRAS, 417, 32 Law, N. F., \& Lane, R. G. 1996, OptCo, 126, 19

Law, N. M., Mackay, C. D., Dekany, R. G., et al. 2009, ApJ, 692, 924

Lloyd, J. P., Martinache, F., Ireland, M. J., et al. 2006, ApJL, 650, L131

MacMartin, D. G. 2003, JOSAA, 20, 1084

Marechal, A. 1947, Rev. d'Opt., 26, 257

Marois, C., Macintosh, B., Barman, T., et al. 2008, Sci, 322, 1348

Mawet, D., Absil, O., Delacroix, C., et al. 2013, A\&A, 552, L13

Mawet, D., Pueyo, L., Lawson, P., et al. 2012, Proc. SPIE, 8442, 844204

Mawet, D., Pueyo, L., Moody, D., Krist, J., \& Serabyn, E. 2010, Proc. SPIE, 7739, 773914 
McBride, J., Graham, J. R., Macintosh, B., et al. 2011, PASP, 123, 692

Metchev, S. A., \& Hillenbrand, L. A. 2009, ApJS, 181, 62

Nakajima, T., Oppenheimer, B. R., Kulkarni, S. R., et al. 1995, Natur, 378, 463

Oppenheimer, B. R., Baranec, C., Beichman, C., et al. 2013, ApJ, 768, 24

Oppenheimer, B. R., Beichman, C., Brenner, D., et al. 2012, Proc. SPIE, 8447, 844720

Oppenheimer, B. R., Dekany, R. G., Hayward, T. L., et al. 2000, Proc. SPIE, 4007, 899

Oppenheimer, B. R., Palmer, D., Dekany, R. G., et al. 1997, Proc. SPIE, 3126,569

Poyneer, L. A., \& Macintosh, B. 2004, JOSAA, 21, 810

Poyneer, L. A., Troy, M., Macintosh, B., \& Gavel, D. T. 2003, OptL, 28, 798

Ren, H., Dekany, R., \& Britton, M. 2005, ApOpt, 44, 2626

Roberts, J., Bouchez, A. H., Burruss, R. S., et al. 2010, Proc. SPIE, 7736, $77362 \mathrm{E}$

Roberts, J. E., Bouchez, A. H., Angione, J., et al. 2008, Proc. SPIE, 7015, $70152 \mathrm{~S}$

Roberts, J. E., Dekany, R. G., Burruss, R. S., et al. 2012, Proc. SPIE, 8447, 84470
Roberts, L. C., Jr., Perrin, M. D., Marchis, F., et al. 2004, Proc. SPIE, 5490, 504 Rousset, G., Lacombe, F., Puget, P., et al. 2003, Proc. SPIE, 4839, 140 Schödel, R., Yelda, S., Ghez, A., et al. 2013, MNRAS, 429, 1367

Serabyn, E., Mawet, D., Bloemhof, E., et al. 2009, ApJ, 696, 40

Shi, F., MacMartin, D. G., Troy, M., et al. 2003, Proc. SPIE, 4839, 1035

Swartzlander, G. A., Jr., Ford, E. L., Abdul-Malik, R. S., et al. 2008, OExpr, 16,10200

Tecza, M., Thatte, N., Clarke, F., et al. 2012, Proc. SPIE, 8446, 844622

Thatte, N., Tecza, M., Clarke, F., et al. 2010, Proc. SPIE, 7735, 77352

Troy, M., Dekany, R. G., Brack, G., et al. 2000, Proc. SPIE, 4007, 31

Truong, T., Brack, G. L., Troy, M., et al. 2003, Proc. SPIE, 4839, 911

Truong, T. N., Bouchez, A. H., Dekany, R. G., et al. 2008, Proc. SPIE, 7015 70153

Velur, V., Flicker, R. C., Platt, B. C., et al. 2006, Proc. SPIE, 6272, 62725

Velur, V., Kibblewhite, E. J., Dekany, R. G., et al. 2004, Proc. SPIE, 5490, 1033

Wyatt, M. C. 2008, ARA\&A, 46, 339

Wyatt, M. C., Dermott, S. F., Telesco, C. M., et al. 1999, ApJ, 527, 918

Zimmerman, N., Brenner, D., Oppenheimer, B. R., et al. 2011, PASP, 123, 746 